PROCEEDINGS OF THE

AMERICAN MATHEMATICAL SOCIETY

Volume 126, Number 10, October 1998, Pages 2855-2856

S $0002-9939(98) 04564-\mathrm{X}$

\title{
NONCOMPLETE LINEAR SYSTEMS ON ELLIPTIC CURVES AND ABELIAN VARIETIES: ADDENDUM TO A PAPER BY CH. BIRKENHAKE
}

\author{
E. BALLICO
}

(Communicated by Ron Donagi)

\begin{abstract}
Here we give a result on the postulation (i.e. the 2-normality) of nonlinearly normal embeddings of Abelian varieties. This result improves some of the results proved in a recent paper by $\mathrm{Ch}$. Birkenhake.
\end{abstract}

In [Bi1], [Bi2] and [Bi3], Ch. II, Ch. Birkenhake considered the postulation and the minimal free resolution of noncomplete embeddings into $\mathbf{P}^{N}$, respectively of Abelian varieties, projective spaces and curves. In [Bi1], $\S 1$ and $\S 2$, a general set-up for the study of the minimal free resolution of noncomplete embeddings of algebraic varieties into $\mathbf{P}^{N}$ was given. A cursory reading of [Bi1] shows that a key point of the proofs was the reduction to the case of a product of elliptic curves and then to the case (via Künneth formula) to the case of an embedding of an elliptic curve. In [Bi1] only the good properties of general projections into $\mathbf{P}^{m-1}$ of a complete embedding into $\mathbf{P}^{m}$ of an elliptic curve were used. However, there are similar results for general projections into $\mathbf{P}^{s}$ with $s$ much smaller than $m-1$ (see [BE1], [BE2], [BE3] and the statement here of 1.1). Here we want to show how to use these results to improve some of the results of [Bi1]. Our result is the following theorem which improves [Bi1], Cor. 4.3.

Theorem 0.1. Suppose $(X, L)$ is a general complex Abelian variety of type $\left(d_{1}, \ldots, d_{g}\right)$. Fix an integer $n \geq 3$ such that $n d_{g} \geq 6$. Let $w$ be the largest integer such that $\left(n d_{g}-w\right)\left(n d_{g}-w+1\right) \geq 4 n d_{g}$. Fix an integer $c$ with $1 \leq c \leq w n^{g-1}$. Then the general vector subspace $V \subseteq H^{0}\left(X, L^{\otimes n}\right)$ with $\operatorname{codim}(V)=c$ is 2-normal, i.e. the restriction map $S^{2}(V) \rightarrow H^{0}\left(X, L^{\otimes 2 n}\right)$ is surjective.

For the postulation of general projections of Hirzebruch surfaces and Veronese embeddings of $\mathbf{P}^{2}$, see [BE4].

\section{THE PROOFS}

As in [Bi1] we work in characteristic 0 . Let $k \geq 1$ be an integer. Recall that with the terminology of [Bi1] a closed subscheme $Z$ of $\mathbf{P}\left(V^{*}\right)$ is $k$-normal if the canonical map $S^{k}(V) \rightarrow H^{0}\left(Z, \boldsymbol{O}_{Z}(k)\right)$ is surjective. The following result was proved in [BE1] and it is a very particular case of the particular case " $g=1$ " of [BE2] (projections into $\mathbf{P}^{3}$ ) and [BE3], Th. I (projections into $\mathbf{P}^{N}, N \geq 4$ ).

Received by the editors March 18, 1997.

1991 Mathematics Subject Classification. Primary 14C20, 14H52, 14 K05.

The author was partially supported by MURST and GNSAGA of CNR (Italy).

(C)1998 American Mathematical Society 
Theorem 1.1. Fix integers $d, N$ with $d>N \geq 3$ and $(N+2)(N+1) / 2 \geq 2 d$. Fix an elliptic curve $Y$ and $L \in \operatorname{Pic}^{d}(Y)$ and let $X \subset \mathbf{P}^{d-1}$ be the linearly normal embedding of $Y$ determined by $H^{0}(Y, L)$. Then the general projection of $X$ into $\mathbf{P}^{N}$ is $k$-normal for all integers $k \geq 2$.

Remark 1.2. Note that since $h^{0}\left(\mathbf{P}^{N}, \boldsymbol{O}_{\mathbf{P}^{N}}(2)\right)=(N+2)(N+1) / 2$ and $h^{0}\left(Y, L^{\otimes 2}\right)=$ $2 d$, the statement of Theorem 1.1 is sharp.

Remark 1.3. Note that in the statement of Theorem 1.1 we do not need the assumption that $L$ is a square, i.e. that $d$ is even, made in [Bi1], Cor. 4.2. Hence in our improvement 0.1 of [Bi1], Cor. 4.3, we do not need the assumption that $n d_{g}$ is even.

Proof of Theorem 0.1. Theorem 0.1 follows from the proof of [Bi1], Cor. 4.3, using 1.1 instead of [Bi1], Cor. 4.2.

\section{REFERENCES}

[BE1] E. Ballico, Ph. Ellia, Sur la postulation des courbes de $\mathbf{P}^{n}$ et de leurs projections, C. R. Acad. Sc. Paris 299 (1984), 237-240. MR 86a:14029

[BE2] E. Ballico, Ph. Ellia, On the projection of a general curve in $\mathbf{P}^{3}$, Annali Mat. Pura e Applicata (4) 142 (1985), 15-48. MR 87g:14026

[BE3] E. Ballico, Ph. Ellia, On the postulation of a general projection of a curve in $\mathbf{P}^{N}, N \geq 4$, Annali Mat. Pura e Applicata (4) 147 (1987), 267-301. MR 88i:14026

[BE4] E. Ballico, Ph. Ellia, On projections of ruled and Veronese surfaces, J. of Algebra 121 (1989), 477-487. MR 90f:14016

[Bi1] Ch. Birkenhake, Linear systems on projective spaces, Manuscripta Math. 88 (1995), 177184. MR 96h: 14003

[Bi2] Ch. Birkenhake, Noncomplete linear systems on abelian varieties, Trans. Amer. Math. Soc. 348 (1995), 1885-1908. MR 97a:14005

[Bi3] Ch. Birkenhake, Nicht volloëindige Liniarsysteme, Habilitaitionsschrift, Erlangen, 1994.

Department of Mathematics, University of Trento, 38050 Povo (TN), Italy

E-mail address: ballico@science.unitn.it 